\title{
X-ray impact on the protoplanetary disks around T Tauri stars
}

\author{
G. Aresu ${ }^{1}$, I. Kamp ${ }^{1}$, R. Meijerink ${ }^{2}$, P. Woitke ${ }^{3,4,5}$, W.-F. Thi ${ }^{5,6}$, and M. Spaans ${ }^{1}$
}

\author{
${ }^{1}$ Kapteyn Astronomical Institute, Postbus 800, 9700 AV Groningen, The Netherlands \\ e-mail: giambattista.aresu@gmail.com \\ 2 Leiden Observatory, Leiden University, PO Box 9513, 2300 RA, Leiden, The Netherlands \\ 3 UK Astronomy Technology Center, Royal Observatory, Edinburgh, Blackford Hill, Edinburgh EH9 3HJ, UK \\ ${ }^{4}$ Institute for Astronomy, University of Edinburgh, Royal Observatory, Blackford Hill, Edinburgh EH9 3HJ, UK \\ ${ }^{5}$ Laboratoire d'Astrophysique de Grenoble, CNRS/Université Joseph Fourier (UMR 5571), BP 53, 38041 Grenoble Cedex 9, France \\ ${ }^{6}$ SUPA, Institute for Astronomy, University of Edinburgh, Royal Observatory Edinburgh, UK
}

Received 22 July 2010 / Accepted 24 November 2010

\section{ABSTRACT}

\begin{abstract}
Context. T Tauri stars have X-ray luminosities in the range $L_{\mathrm{X}}=10^{28}-10^{32} \mathrm{erg} \mathrm{s}^{-1}$. These luminosities are similar to their UV luminosities $\left(L_{\mathrm{UV}} \sim 10^{30}-10^{31} \mathrm{erg} \mathrm{s}^{-1}\right)$ and therefore X-rays are expected to affect the physics and chemistry of the upper layers of their surrounding protoplanetary disks.

Aims. The effects and importance of X-rays on the chemical and hydrostatic structure of protoplanetary disks are investigated, species tracing X-ray irradiation (for $L_{\mathrm{X}} \geq 10^{29} \mathrm{erg} \mathrm{s}^{-1}$ ) are identified and predictions for [O I], [C II], and [N II] fine structure line fluxes are provided.

Methods. We implemented X-ray physics and chemistry into the chemo-physical disk code ProDiMo. We include Coulomb heating and $\mathrm{H}_{2}$ ionization as heating processes and both primary and secondary ionization due to X-rays in the chemistry.

Results. X-rays heat the gas causing it to expand in the optically thin surface layers. Neutral molecular species are not significantly affected in terms of their abundance and spatial distribution, but charged species such as $\mathrm{N}^{+}, \mathrm{OH}^{+}, \mathrm{H}_{2} \mathrm{O}^{+}$, and $\mathrm{H}_{3} \mathrm{O}^{+}$display enhanced abundances in the disk surface.

Conclusions. Coulomb heating by X-rays changes the vertical structure of the disk, yielding temperatures of $\sim 8000 \mathrm{~K}$ out to distances of $50 \mathrm{AU}$. The chemical structure is altered by the high electron abundance of the gas at the disk surface, causing an efficient ion-molecule chemistry. The products of this, $\mathrm{OH}^{+}, \mathrm{H}_{2} \mathrm{O}^{+}$and $\mathrm{H}_{3} \mathrm{O}^{+}$, are of great interest for observations of low-mass young stellar objects with the Herschel Space Observatory. Both [O I] (at 63 and $145 \mu \mathrm{m}$ ) and [C II] (at $158 \mu \mathrm{m}$ ) fine structure emission are affected only for $L_{\mathrm{X}}>10^{30} \mathrm{erg} \mathrm{s}^{-1}$.
\end{abstract}

Key words. protoplanetary disks - methods: numerical

\section{Introduction}

Young stellar objects emit X-ray radiation (Koyama et al. 1994), which is associated with magnetic coronal processes (Flaccomio et al. 2005; Wolk et al. 2005; Imanishi et al. 2001), jets (Pravdo et al. 2001) mostly producing soft X-rays (Güdel et al. 2007), or outflows launched in the region between the star and the disk as predicted by the X-wind model (Shu et al. 2000). Non-accreting weak-line T Tauri stars (WTTS) are found to be more X-ray luminous than classical accreting T Tauri stars (CTTS) (Stelzer \& Neuhäuser 2001; Flaccomio et al. 2003, 2005), because CTTS absorb part of the X-rays in their accretion column (Gregory et al. 2007).

The impact of stellar X-rays on protoplanetary disks around $\mathrm{T}$ Tauri stars has been studied by many groups. Glassgold et al. $(2004,2007)$ found X-rays to be important in heating the surface layers of the disk and estimated the strength of the finestructure emission lines of [Ne II] and [Ne III], which arise from the warm disk atmosphere exposed to X-ray radiation. Nomura et al. (2007) focused on $\mathrm{H}_{2}$ level populations and line emission, finding $\mathrm{X}$-rays to control $\mathrm{H}_{2}$ level population pumping together with UV. Following the work of Meijerink et al. (2008), Ercolano et al. (2008) and Glassgold et al. (2009) studied the atomic-line diagnostics of the inner regions of protoplanetary disks and formation of water in the warm disk atmosphere, respectively. Ercolano et al. (2008) and others focused on the
X-ray role in causing the photo-evaporation of the surface layers of the disk. Gorti \& Hollenbach $(2004,2008)$ include X-rays to predict [Ar II, [Ne II], [Fe I], [S I], [Fe II], and [Si II] as good indicators of gas physics in the disk. Woods \& Willacy (2009) studied carbon isotope fractionation using the same method as Gorti \& Hollenbach (2004) to calculate the X-ray ionization rates. Henning et al. (2010) studied the role of UV and X-rays on $\mathrm{C}_{2} \mathrm{H}$ column densities and the excitation conditions in disks around $\mathrm{T}$ Tauri stars.

The scope of this paper is to take a step back and first perform a study of the impact of X-rays on the 2D disk structure, on molecular ionized species, and on observational diagnostics such as [OI], [C II], and [NII]. These molecular species were not studied in the above listed previous papers, but are of fundamental interest in the light of their detections with the Herschel satellite (Benz et al. 2010; Bruderer et al. 2010). We undertake an exploratory study of the relative effects of UV and X-rays on the protoplanetary disks hydrostatic, thermal, and chemical structure using a series of X-ray models with different $L_{\mathrm{X}}$ (X-ray luminosity).

\section{Model}

The chemo-physical disk modeling code ProDiMo (Woitke et al. 2009; Kamp et al. 2010) has been updated with X-ray physics and chemistry. 
Table 1. Examples of molecular dissociation and cross-section calculation.

\begin{tabular}{llll}
\hline \hline Molecule & Product 1 & Product 2 & Cross-section \\
\hline $\mathrm{CO}$ & $\mathrm{C}^{2+}$ & $\mathrm{O}$ & $\sigma_{\mathrm{CO}}=1 / 3 \sigma_{\mathrm{C}}+$ \\
& $\mathrm{C}^{+}$ & $\mathrm{O}^{+}$ & $1 / 3\left(0.5 \sigma_{\mathrm{C}}+0.5 \sigma_{\mathrm{O}}\right)+$ \\
& $\mathrm{C}$ & $\mathrm{O}^{2+}$ & $1 / 3 \sigma_{\mathrm{O}}$ \\
\hline $\mathrm{CH}$ & $\mathrm{C}^{2+}$ & $\mathrm{H}$ & $\sigma_{\mathrm{CH}}=\sigma_{\mathrm{C}}$ \\
\hline
\end{tabular}

Notes. $\mathrm{CO}$ dissociation can end up in three different channels, which are summed to give the total $\mathrm{CO}$ cross-section, $\mathrm{CH}$ is only assumed to follow one path. Molecules included: $\mathrm{H}_{2}, \mathrm{CH}, \mathrm{NH}, \mathrm{OH}, \mathrm{CN}, \mathrm{CO}, \mathrm{N}_{2}$, $\mathrm{SiH}, \mathrm{NO}, \mathrm{O}_{2}, \mathrm{SiO}, \mathrm{CH}_{2}, \mathrm{NH}_{2}, \mathrm{H}_{2} \mathrm{O}, \mathrm{HCN}, \mathrm{CO}_{2}$.

\subsection{Input spectrum}

The incident stellar spectrum used in Woitke et al. (2009) is composed of a solar model with $T_{\text {eff }}=5800 \mathrm{~K}$ and the cromospheric fluxes of HD 129333 (Dorren \& Guinan 1994). The UV luminosity between 91.2 and $205 \mathrm{~nm}$ is $L_{\mathrm{UV}} \sim 4 \times 10^{31} \mathrm{erg} \mathrm{s}^{-1}$. Different models for the X-ray input spectrum are adopted in the literature: Nomura et al. (2007) use a two-temperature thin thermal-plasma model to fit the observed TW Hydrae spectrum; Ercolano et al. (2008) compiled synthetic coronal spectra using line and continuum emissivities from the CHIANTI compilation of atomic data; Gorti \& Hollenbach (2008) use a thermal blackbody. We place the X-ray source on the star and follow Glassgold et al. (1997) and Igea \& Glassgold (1999) in using an analytic input spectrum to describe the bremsstrahlung emission from an isothermal plasma $F(E) \propto 1 / E \cdot \exp \left(-E / k T_{\mathrm{X}}\right)$ where $E$ is the photon energy between 0.1 and $100 \mathrm{keV}, k T_{\mathrm{X}}=1 \mathrm{keV}$, and $T_{\mathrm{X}}$ is the plasma temperature. The X-ray input spectrum is added to the input spectrum shown in Fig. 2 in Woitke et al. (2009).

\subsection{X-ray chemistry}

The following paragraphs describe the implementation of primary and secondary ionization in the code. Forty-one primary ionization reactions and 16 secondary ionization reactions were added to the chemical network.

\section{Primary ionization}

The primary rates for X-ray absorption are calculated following Meijerink \& Spaans (2005) (Appendix D.3.1). The crosssections are taken from Verner \& Yakovlev (1995). Since X-rays are likely to be absorbed in the K-shells, we assume that every $\mathrm{X}$-ray absorption leads to a single ionization for $\mathrm{H}, \mathrm{He}, \mathrm{Si}$, and $\mathrm{Cl}$ and a double ionization for $\mathrm{C}, \mathrm{N}, \mathrm{O}, \mathrm{S}$, and $\mathrm{Fe}$ (Meijerink $\&$ Spaans 2005). We take into account molecular X-ray absorption, which always leads to dissociation, of the species. Table 1 lists two examples of how the total dissociation rates are calculated. When the difference in weight of the elements that form the molecule is large (e.g. $\mathrm{CH}, \mathrm{OH}, \mathrm{H}_{2} \mathrm{O}$ etc.), we use the crosssection of the heavier element, when the weight is comparable (e.g. $\mathrm{CO}, \mathrm{CN}, \mathrm{NO}$ etc.) we combine the cross-sections of the single elements.

\section{Secondary ionization}

The fast electrons generated by the X-ray absorption can further ionize the gas. The rates are computed as shown in Meijerink \& Spaans (2005, Appendix D.3.2) using experimental data from Lennon et al. (1988). The volumetric rates are a function of the
Table 2. Parameters used in the models: the X-ray luminosity values correspond to the models 1-5.

\begin{tabular}{lcc}
\hline \hline Quantity & Symbol & Value \\
\hline Stellar mass & $M_{*}$ & $1 M_{\odot}$ \\
Stellar luminosity & $L_{*}$ & $1 L_{\odot}$ \\
Disk mass & $M_{\text {disk }}$ & $0.01 M_{\odot}$ \\
X-ray luminosity $(0.1-50 \mathrm{keV})$ & $L_{\mathrm{X}}$ & $0,10^{29}, 10^{30}$ \\
& & $10^{31}, 10^{32}$ \\
Inner disk radius & $R_{\text {in }}$ & $0.5 \mathrm{AU}$ \\
Outer disk radius & $R_{\text {out }}$ & $500 \mathrm{AU}$ \\
Surface density power law index & $\epsilon$ & 1.5 \\
Min. dust particle size & $a_{\min }$ & $0.1 \mu \mathrm{m}$ \\
Max. dust particle size & $a_{\max }$ & $10 \mu \mathrm{m}$ \\
Dust size distribution power index & $a_{\text {pow }}$ & 2.5 \\
\hline
\end{tabular}

local chemistry, i.e. they depend on the species densities $n_{\mathrm{H}}, n_{\mathrm{H}_{2}}$, $n_{\langle\mathrm{H}\rangle}$, and $n_{\mathrm{e}}$ (where $n_{H}$ is the hydrogen atom density and $n_{\langle\mathrm{H}\rangle}$ is the hydrogen nuclei density). This has to be taken into account when solving the chemical equilibrium. The additional entries in the chemical Jacobian that come from the X-ray secondary ionization reactions are implemented following Sect. 5.6 in Woitke et al. (2009).

\subsection{X-ray heating}

We added Coulomb heating (Dalgarno et al. 1999) and $\mathrm{H}_{2}$ ionization heating (Meijerink \& Spaans 2005, Appendix B.1) to the heating processes listed in Woitke et al. (2009). The Maloney et al. (1996) heating rate holds only for gas with low $x_{\mathrm{e}}$, while Dalgarno et al. (1999) present the more general case for different high and low ionization gases (atomic/molecular). The heating efficiency increases by a factor of 7-8 in a highly ionized atomic gas and a factor of 2 in a highly ionized molecular gas (Shull \& van Steenberg 1985).

\subsection{Parameter space}

We computed five different models with increasing $L_{X}$ to compare the results with the UV only case (model 1 ) for a disk surrounding a $\mathrm{T}$ Tauri star (Table 2 ). In model $1, \mathrm{X}$-rays are switched off. This is the model presented in Woitke et al. (2009). For more details, we refer to the original paper. X-ray models (model 2-5) have the same parameters as model 1 , but include stellar X-rays $\left(L_{\mathrm{X}}=10^{29}-10^{32} \mathrm{erg} \mathrm{s}^{-1}\right)$. We added $\mathrm{Cl}, \mathrm{Cl}^{+}$, and double ionized species $\left(\mathrm{C}^{2+}, \mathrm{N}^{2+}, \mathrm{O}^{2+}, \mathrm{S}^{2+}, \mathrm{Fe}^{2+}\right)$ to the chemical network. The X-ray emission is treated as a point source at the location of the star.

\section{Results}

We analyze the results obtained from the five models described above. We first present the thermal and hydrostatic structure of the various models. We then describe the impact of X-rays on the chemistry and fine-structure line emission, focusing on the main differences between the UV-only model and the combined $\mathrm{UV}+\mathrm{X}$-ray models. 
G. Aresu et al.: X-ray impact on the protoplanetary disks around T Tauri stars
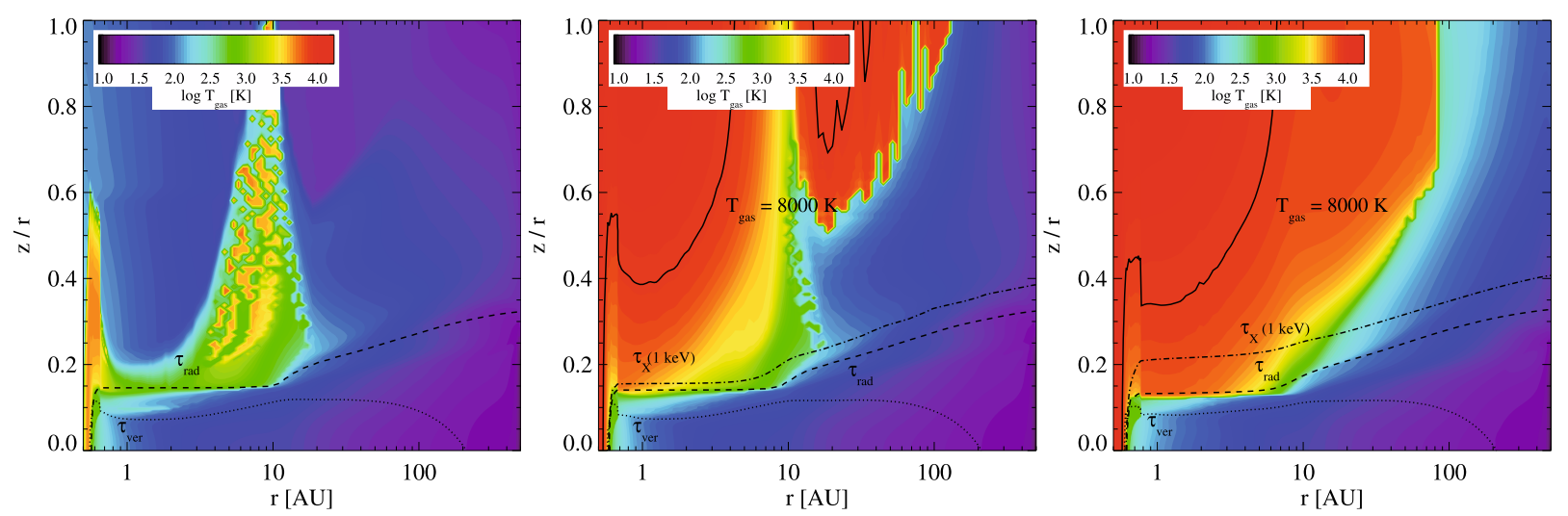

Fig. 1. Gas temperature distribution: Model 1 (UV only) in the left panel, X-ray models with $L_{\mathrm{X}}=10^{30} \mathrm{erg} \mathrm{s}^{-1}(\operatorname{model} 3), L_{\mathrm{X}}=10^{32} \mathrm{erg} \mathrm{s}^{-1}$ (model 5) in the middle and right panel, respectively. Contour lines are plotted over for $\tau_{\text {rad }}=1$ (radial dust optical depth at $550 \mathrm{~nm}$, dashed line), $\tau_{\text {ver }}=1$ (vertical dust optical depth at $550 \mathrm{~nm}$, dotted line), and an X-ray optical depth of one at $1 \mathrm{keV}$ (dot-dashed line). The relative positions of these two depths depend strongly on the assumed dust properties (see Table 2). The solid line corresponds to $T_{\text {gas }}=8000 \mathrm{~K}$.
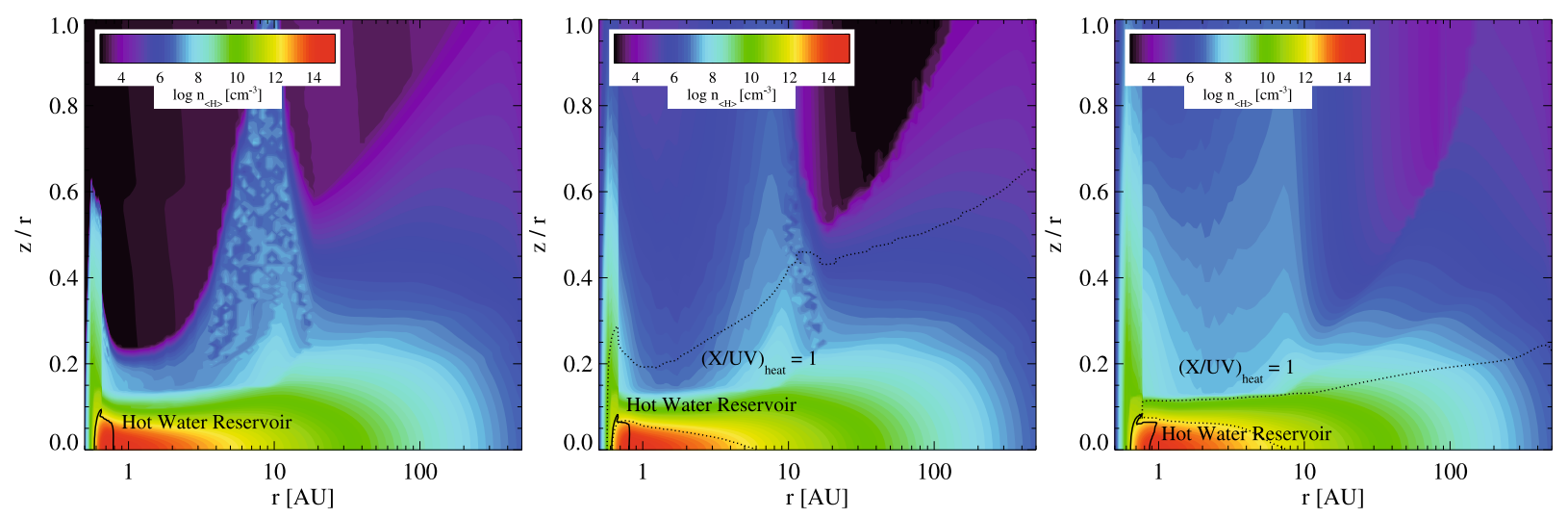

Fig. 2. Total hydrogen nuclei density $n_{\langle\mathrm{H}\rangle}$ distribution: Model 1 (UV only) in the left panel, X-ray models with $L_{\mathrm{X}}=10^{30} \mathrm{erg} \mathrm{s}^{-1}$ (model 3 ), $L_{\mathrm{X}}=10^{32} \mathrm{erg} \mathrm{s}^{-1}$ (model 5) in the middle and right panels, respectively. The dotted contour line shows where the ratio of Coulomb heating to photo-electric plus PAH heating equals 1 . The solid line encircles the hot water reservoir $\left(T_{\mathrm{H}_{2} \mathrm{O}}>200 \mathrm{~K}\right)$.

\section{Temperature and density structure}

The low density $\left(n_{\langle\mathrm{H}\rangle}<10^{8} \mathrm{~cm}^{-3}\right)$ surface layers directly exposed to $\mathrm{X}$-ray radiation are heated efficiently by means of Coulomb heating: in all X-ray models, temperatures reach $\sim 8000 \mathrm{~K}$ (Fig. 1). The extension of this high temperature region increases with $L_{\mathrm{X}}$ : it reaches $2 \mathrm{AU}$ in model 2 and $100 \mathrm{AU}$ in model 3 and 4 . In model 5, the temperature is slightly lower for $r>50 \mathrm{AU}$ but the whole disk is warmer (Fig. 1). X-rays dominate the thermal balance only in the upper layers down to $(\mathrm{X} / \mathrm{UV})_{\text {heat }}=1$. Beyond $(\mathrm{X} / \mathrm{UV})_{\text {heat }}=1$, UV heating takes over and becomes the main heating process. Deeper within the midplane, both X-ray and UV heating are negligible, but their ratio is again inverted and harder X-rays $(E>10 \mathrm{keV})$ deposit more energy than UV radiation (Fig. 2).

The inner wall of the disk is always directly illuminated by the stellar radiation causing the inner rim $(0.5 \mathrm{AU}<r<0.7 \mathrm{AU})$ to have a large scale-height and hence vertical extent. In the UV model, the shadowed disk atmosphere beyond the inner rim has a lower vertical scale-height since radiation cannot penetrate efficiently and sustain high gas temperatures. Only radiation that impinges the disk at higher angles $(z / r>0.6)$ and scattered radiation reach the outer disk causing the temperature to increase and consequently the production of a second bump at $\sim 9$ AU. When $\mathrm{X}$-rays are switched on, the temperature increase in the surface layers directly affects the vertical density distribution there since we keep the radial surface density profile fixed. The inner rim of the disk ( $r<1 \mathrm{AU}$ and $z / r>0.1)$ is more puffed up (Fig. 2). The second bump at $\sim 9$ AU is also more vertically extended; in model 5, it even "merges" with the inner rim and flares as a whole (Fig. 2, third panel).

\section{Chemistry}

The abundances of neutral molecules such as $\mathrm{CO}, \mathrm{OH}$, and $\mathrm{H}_{2} \mathrm{O}$ generally change by less than an order of magnitude over the entire modeling space. On the other hand, the ion chemistry in the disk surface strongly changes: the electron abundance in the upper layers increases by a factor of $10^{4}$. This leads to an enhancement in the $\mathrm{OH}^{+}, \mathrm{H}_{2} \mathrm{O}^{+}, \mathrm{H}_{3} \mathrm{O}^{+}$, and $\mathrm{N}^{+}$abundances. Secondary ionization of $\mathrm{H}_{2}$ increases the $\mathrm{H}_{2}^{+}$abundances. $\mathrm{H}_{2}^{+}$collisions with $\mathrm{H}_{2}$ produce $\mathrm{H}_{3}^{+}$. Subsequent collisions of $\mathrm{H}_{3}^{+}$with $\mathrm{O}, \mathrm{OH}$, and $\mathrm{H}_{2} \mathrm{O}$ lead to the production of $\mathrm{OH}^{+}, \mathrm{H}_{2} \mathrm{O}^{+}$, and $\mathrm{H}_{3} \mathrm{O}^{+}$, respectively. Figure 4 shows how the vertical column densities of these species change with $L_{X}$ for three different disk radii: 3, 10, and $100 \mathrm{AU}$. Both $\mathrm{OH}^{+}$and $\mathrm{H}_{2} \mathrm{O}^{+}$respond more efficiently than $\mathrm{H}_{3} \mathrm{O}^{+}$to the $\mathrm{X}$-ray radiation as the total mass ratio $\mathrm{M}\left(\mathrm{OH}^{+}\right) / \mathrm{M}\left(\mathrm{H}_{2} \mathrm{O}^{+}\right)$only doubles from model 1 to model 5 , while $\mathrm{M}\left(\mathrm{OH}^{+}\right) / \mathrm{M}\left(\mathrm{H}_{3} \mathrm{O}^{+}\right)$increases by more than a factor of 5 .

$\mathbf{O H}^{+}$: $\mathrm{OH}^{+}$formation is enhanced approximately proportionally to the X-ray luminosity. Its total mass increases from $8 \times 10^{-15} M_{\odot}$ in model 1 by a factor of 3.6 in model 3 to about a factor of 500 in model 5 (Table 4). 
Table 3. Predicted line fluxes of [O I] at $63 \mu \mathrm{m}$, [C II] at $157 \mu \mathrm{m}$ and $[\mathrm{N} \mathrm{II}]$ at $205 \mu \mathrm{m}$ for all the models expressed in $\mathrm{W} / \mathrm{m}^{2}$.

\begin{tabular}{llllll}
\hline \hline Line & Model 1 & Model 2 & Model 3 & Model 4 & Model 5 \\
\hline [OI] $63 \mu \mathrm{m}$ & $4.8(-17)$ & $4.6(-17)$ & $4.8(-17)$ & $8.3(-17)$ & $3.6(-16)$ \\
[O I $145 \mu \mathrm{m}$ & $1.5(-18)$ & $1.3(-18)$ & $1.4(-18)$ & $2.3(-18)$ & $1.2(-17)$ \\
[C II $157 \mu \mathrm{m}$ & $1.5(-17)$ & $1.5(-17)$ & $1.7(-17)$ & $3.0(-17)$ & $6.1(-17)$ \\
[N II] $205 \mu \mathrm{m}$ & $3.6(-26)$ & $5.2(-23)$ & $4.6(-22)$ & $4.1(-21)$ & $1.1(-20)$ \\
\hline
\end{tabular}

Notes. All fluxes are computed for a distance of $140 \mathrm{pc}$.

As the X-ray luminosity increases, $\mathrm{OH}^{+}$formation is enhanced and pushed outwards, where the disk is colder. The column density of $\mathrm{OH}^{+}$increases substantially beyond $r>10 \mathrm{AU}$ in all models. It reaches $\sim 100$ times its initial value (model 1 ) in model 3 at $100 \mathrm{AU}$ (Fig. 4).

$\mathrm{H}_{2} \mathrm{O}^{+}$: The $\mathrm{H}_{2} \mathrm{O}^{+}$mass increases by a factor of 3 (a factor of 100) between model 1 and model 3 (and Model 5). The column density again increases toward the outer part of the disk. Model 3 has $\sim 10$ times higher column density than Model 1 at both 10 and $100 \mathrm{AU}$. As for $\mathrm{OH}^{+}, \mathrm{H}_{2} \mathrm{O}^{+}$appears to move toward the outer part of the disk with increasing $\mathrm{X}$-ray luminosity.

$\mathbf{H}_{3} \mathrm{O}^{+}$: The $\mathrm{H}_{3} \mathrm{O}^{+}$mass is slightly less affected by X-rays: it is two times higher than in the UV case in model 3 and ten times in model 4. In model 3 , model 4 , and model 5 , the region of high $\mathrm{H} 3 \mathrm{O}+$ abundances is also clearly pushed to larger radii and hence the species mass averaged temperature becomes lower.

$\mathrm{N}^{+}$: We find the most extreme mass increase for $\mathrm{N}^{+}$in all X-ray models (Table 4 ) and at all radii: in model 3 , it is 1000 times higher than in the UV-only case.

In models 4 and 5 , the $\mathrm{N}^{+}$mass increases overproportionally in the outer part of the disk (Fig. 4, right lower panel). Figure 5 shows the column density ratio of the $\mathrm{X}$-ray dominated $\mathrm{N}^{+}$to the $\mathrm{UV}$ dominated $\mathrm{C}^{+}$. With increasing $L_{\mathrm{X}}$, the $\mathrm{N}^{+}$column density is enhanced by two (outer disk) to six (inner disk) orders of magnitude.

\section{Line predictions}

In Table 3, we list the flux prediction of [OI] $63 \mu \mathrm{m}$, [OI] $145 \mu \mathrm{m}$, [C II] $157 \mu \mathrm{m}$, and [N II] $205 \mu \mathrm{m}$ for all the models. Figure 3 shows their values as a function of the X-ray luminosity. The [N II] line flux shows the strongest correlation with the X-ray flux, increasing by five orders of magnitude from model 1 to model 3. The oxygen fine-structure line fluxes and the [C II] line flux are constant from model 1 to 3 (Fig. 3), because they originate in the UV-heated layer. Only in models 4 and 5 do the line fluxes increase with $L_{\mathrm{X}}$.

\section{Discussions and conclusions}

We have found that X-rays mainly impact the surface layers of protoplanetary disks. The hydrostatic structure of these layers is changed significantly: $\mathrm{X}$-ray radiation is mostly absorbed in the tenuous layers $\left(z / r \geq 0.2 \mathrm{~cm}^{-3}\right)$ increasing the temperature to $\sim 8000 \mathrm{~K}$; at those temperatures, cooling by [O I], Fe II, and Ly $\alpha$ balance the X-ray heating. This causes the density structure to flare more strongly than in the UV-only case (Fig. 2).

The general results of Nomura et al. (2007) and Glassgold et al. (2004) are qualitatively confirmed, showing that the inner rim and the surface layers are dominated by X-rays. In addition, we have found that the more distant parts, out to $100 \mathrm{AU}$, are also affected (Fig. 4). We find that the size of the high temperature

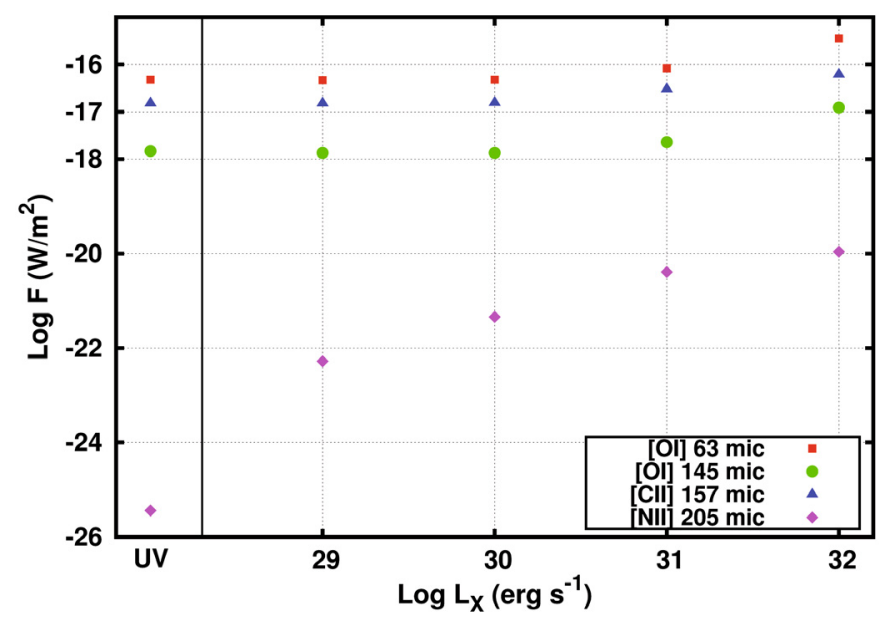

Fig. 3. Oxygen-line flux emission at 63 and $157 \mu \mathrm{m}$, ionized carbonline flux emission at $157 \mu \mathrm{m}$ and ionized nitrogen-line flux emission at $205 \mu \mathrm{m}$ as a function of the X-ray luminosity. All fluxes are compiled for a distance of $140 \mathrm{pc}$. The correlation between N II and $L_{\mathrm{X}}$ is clear, while $\mathrm{O}$ I and C II show no correlation for $L_{\mathrm{X}}<10^{30} \mathrm{erg} \mathrm{s}^{-1}$.

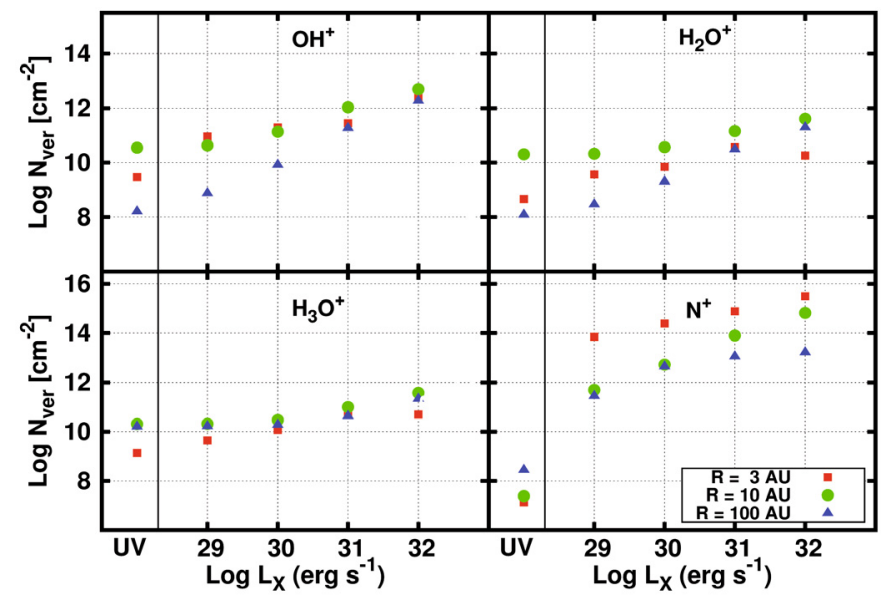

Fig. 4. Column density of $\mathrm{OH}^{+}$(upper left panel), $\mathrm{H}_{2} \mathrm{O}^{+}$(upper right panel), $\mathrm{H}_{3} \mathrm{O}^{+}$(lower left panel), and $\mathrm{N}^{+}$(lower right panel) at three different radii $(3,10,100 \mathrm{AU})$ versus X-ray luminosity. In each panel, the UV-only model is indicated by the "UV" label on the $x$-axis.

X-ray driven region increases with $L_{\mathrm{X}}$. This is because our model uses the Dalgarno et al. (1999) prescription for X-ray heating and of our choice of the input spectrum. The heating efficiencies of Dalgarno et al. (1999) lead to $~ 7-8$ times higher rates in regions with high electron fraction, than Maloney et al. (1996). Furthermore, our input spectrum has higher photon flux in the range $0.1-0.3 \mathrm{keV}$ than, e.g., Gorti \& Hollenbach (2008) and Nomura et al. (2007). These soft X-ray photons are absorbed at low vertical column-densities causing high $\mathrm{e}^{-}$densities there and are thus more efficient in heating the upper layers. This was also noted in Gorti et al. (2009).

Gorti \& Hollenbach (2008) found X-rays to be the dominant heating process in slightly deeper layers than we do. This is most likely because we include UV scattering from dust, which leads to a more substantial vertical penetration of FUV radiation into the disk. Nomura et al. (2007) also show that FUV penetrates deeper than X-rays, because FUV scattering is more efficient than X-ray Compton scattering at $1 \mathrm{keV}$, which is not included in our model. Gorti \& Hollenbach (2008) also consider a less steep 
Table 4. Species masses given in units of solar mass for all the models.

\begin{tabular}{lccccc}
\hline \hline Species & UV Only & $L=10^{29} \mathrm{erg} \mathrm{s}^{-1}$ & $L=10^{30} \mathrm{erg} \mathrm{s}^{-1}$ & $L=10^{31} \mathrm{erg} \mathrm{s}^{-1}$ & $L=10^{32} \mathrm{erg} \mathrm{s}^{-1}$ \\
\hline $\mathrm{H}$ & $5.0(-05)$ & $5.0(-05)$ & $5.2(-05)$ & $7.1(-05)$ & $1.4(-04)$ \\
$\mathrm{H}_{2}$ & $7.6(-03)$ & $7.6(-03)$ & $7.6(-03)$ & $7.6(-03)$ & $7.5(-03)$ \\
$\mathrm{C}$ & $3.0(-07)$ & $3.0(-07)$ & $3.1(-07)$ & $3.4(-07)$ & $4.8(-07)$ \\
$\mathrm{C}+$ & $1.3(-07)$ & $1.3(-07)$ & $1.3(-07)$ & $1.4(-07)$ & $1.8(-07)$ \\
$\mathrm{O}$ & $1.4(-05)$ & $1.4(-05)$ & $1.4(-05)$ & $1.4(-05)$ & $1.4(-05)$ \\
$\mathrm{CO}$ & $9.5(-06)$ & $9.4(-06)$ & $9.3(-06)$ & $9.0(-06)$ & $9.0(-06)$ \\
$\mathrm{OH}$ & $3.4(-11)$ & $3.4(-11)$ & $3.7(-11)$ & $7.0(-11)$ & $2.8(-10)$ \\
$\mathrm{H}{ }_{2} \mathrm{O}$ & $7.7(-07)$ & $4.7(-08)$ & $3.6(-08)$ & $2.7(-08)$ & $3.3(-08)$ \\
$\mathrm{OH}^{+}$ & $7.9(-15)$ & $1.1(-14)$ & $2.9(-14)$ & $4.1(-13)$ & $4.6(-12)$ \\
$\mathrm{H}_{2} \mathrm{O}^{+}$ & $1.9(-15)$ & $2.1(-15)$ & $5.3(-15)$ & $6.2(-14)$ & $4.2(-13)$ \\
$\mathrm{H}_{3} \mathrm{O}^{+}$ & $3.4(-15)$ & $3.7(-15)$ & $7.3(-15)$ & $4.8(-14)$ & $3.5(-13)$ \\
$\mathrm{N}^{+}$ & $2.9(-15)$ & $8.7(-13)$ & $3.9(-12)$ & $9.9(-12)$ & $3.6(-11)$ \\
\hline
\end{tabular}

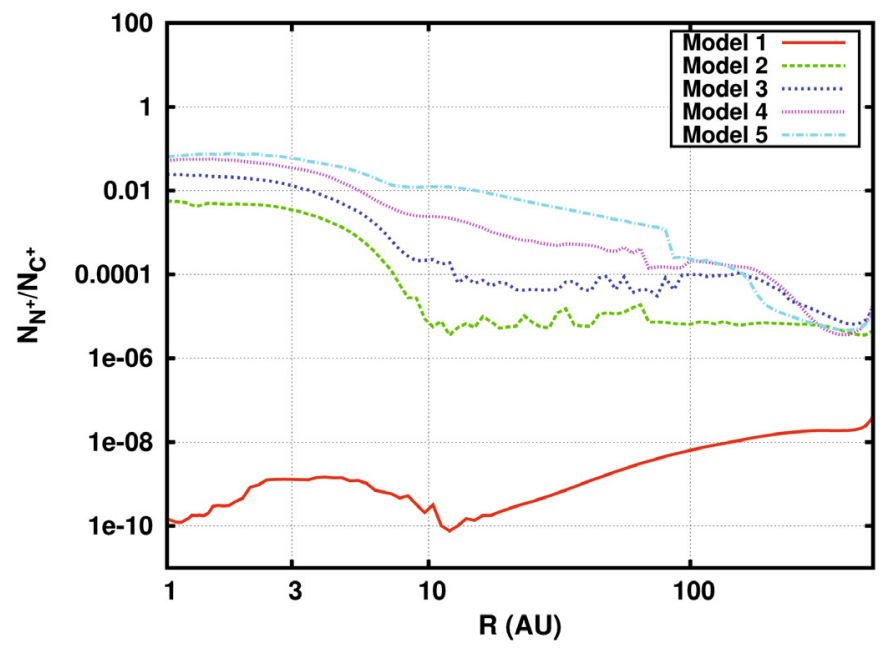

Fig. 5. Column density ratio of the X-ray dominated $\mathrm{N}^{+}$to the UV dominated $\mathrm{C}^{+}$. The latter does not change in the $\mathrm{X}$-ray models with respect to the UV model.

surface-density distribution that yields less mass at $1 \mathrm{AU}$, where most of the X-ray radiation is absorbed in our model.

The X-ray energy deposition does not affect the neutral oxygen fine-structure emission at 63 and $145 \mu \mathrm{m}$ (Table 3), unless the X-ray luminosity is pushed to $L_{\mathrm{X}}>10^{30} \mathrm{erg} \mathrm{s}^{-1}$, where $\mathrm{X}$-ray heating dominates in the regions where the emission takes place. Ionized carbon emission at $157 \mu \mathrm{m}$ is only slightly affected by the X-rays in all the models, as the line emission is dominated by material at $R>200$ AU (Woitke et al. 2009; Kamp et al. 2010), where the X-ray contribution to the chemistry and thermal balance is negligible. Our values are in good agreement with Meijerink et al. (2008) and Ercolano et al. (2008) for $L_{\mathrm{X}} \sim 10^{30} \mathrm{erg} \mathrm{s}^{-1}$. However, the correlation of the oxygen line fluxes with $L_{X}$, found in the Meijerink et al. (2008) model is caused by the lack of UV radiation in their model. In our model at low $L_{X}$, the UV heating ensures that the gas temperature is sufficiently high to sustain a constant level of [O I] emission.

We have found that the $\mathrm{H}_{2}, \mathrm{CO}$, and $\mathrm{OH}$ properties of the $\mathrm{X}$-ray models are largely unvaried with respect to model 1 . The largest change occurs for water, whose mass diminishes by a factor of 20 in the highest $L_{X}$ model relative to the UV-only model. The X-rays enhance the electron abundance in the surface layers, where we find that $x_{\mathrm{e}} \sim 0.1$ for $z / r \sim 0.9$ out to $100 \mathrm{AU}$. Ionized species abundances such as $\mathrm{OH}^{+}, \mathrm{H}_{2} \mathrm{O}^{+}, \mathrm{H}_{3} \mathrm{O}^{+}$, and $\mathrm{N}^{+}$, are enhanced. The molecular ionized species form at increasingly greater radii as $L_{\mathrm{X}}$ increases. High abundances of $\mathrm{OH}^{+}$and $\mathrm{H}_{2} \mathrm{O}^{+}$are a characteristic signature of an X-ray dominated region as discussed by van der Werf et al. (2010) after the detection with Herschel/SPIRE in the ultra-luminous galaxy Mrk 231. Furthermore, this is extremely interesting in the context of the detection of these species in a massive YSO by Herschel (Benz et al. 2010). The column densities that we find from our X-ray irradiated disks are of the same order of magnitude as those derived in Benz et al. (2010). This suggests that a disk could in principle provide a substantial contribution to the line fluxes emitted from these molecules, making our result extremely relevant to future Herschel studies of low-mass YSOs.

The abundance of these ions increases especially at larger radii ( $r>10 \mathrm{AU}$ ), while the $\mathrm{N}^{+}$abundance rises considerably at all radii. The latter is not observed in the UV model because of the high ionization potential of N(I.P.) $=14.5 \mathrm{eV}$. We have found that the $\mathrm{N}^{+}$flux emitted at $205 \mu \mathrm{m}$ in model 3 is $\sim 5 \times 10^{-22} \mathrm{~W} / \mathrm{m}^{2}$, coming for $90 \%$ from the upper layers beyond $10 \mathrm{AU}$. This is much smaller than the current Herschel sensitivity limit of $5 \times 10^{-18} \mathrm{~W} / \mathrm{m}^{2}$ (HIFI, $5 \sigma$ in $1 \mathrm{~h}$ ) and even below current sensitivity estimates for SPICA/SAFARI $\left(10^{-19} \mathrm{~W} / \mathrm{m}^{2}, 5 \sigma\right.$ in $1 \mathrm{~h}$ ). We have only considered X-ray ionization, but the flux is unlikely to change if EUV is present. Additional ionization occurs only at smaller radii, because of the low penetration depth of EUV radiation (Hollenbach \& Gorti 2009). The formation of molecular ionized species moves toward the outer disk as $L_{\mathrm{X}}$ increases.

\section{Outlook}

Our predicted $\mathrm{N}^{+}$line fluxes are too low to be observed with current instruments. In contrast, the [O I] $63 \mu \mathrm{m}$ and [C II] $157 \mu \mathrm{m}$ lines are observable in the presence of UV luminosities of $\sim 10^{31} \mathrm{erg} \mathrm{s}^{-1}$ and/or strong X-rays $\left(L_{\mathrm{X}}>10^{30} \mathrm{erg} \mathrm{s}^{-1}\right)$. In the context of the Herschel observatory, the $\mathrm{OH}^{+}, \mathrm{H}_{2} \mathrm{O}^{+}$, and $\mathrm{H}_{3} \mathrm{O}^{+}$lines are extremely interesting as they are likely to be observable with the HIFI, PACS, and SPIRE instruments. These hydride ions and the already observed [Ne II] and [Ar II] line fluxes, will be addressed in a future paper (Aresu et al., in prep.). We will also investigate a larger parameter space and the effect of the change in disk structure - especially of a higher inner rim and greater flaring - on the overall SED. Future work will also include the creation of a grid of X-ray models following the Woitke et al. (2010) approach.

Acknowledgements. The authors acknowledge the anonymous referee for helpful comments that improved the clarity and completeness of the paper. This work has been funded by The Netherlands Organization for Scientific Research 
(NWO). The LAOG group acknowledge PNPS, CNES, and ANR (contract ANR-07-BLAN-0221) for financial support.

\section{References}

Benz, A. O., Bruderer, S., van Dishoeck, E. F., et al. 2010, A\&A, 521, L35 Bruderer, S., Benz, A. O., van Dishoeck, E. F., et al. 2010, A\&A, 521, L44 Dalgarno, A., Yan, M., \& Liu, W. 1999, ApJS, 125, 237

Dorren, J. D., \& Guinan, E. F. 1994, ApJ, 428, 805

Ercolano, B., Drake, J. J., Raymond, J. C., \& Clarke, C. C. 2008, ApJ, 688, 398

Flaccomio, E., Micela, G., \& Sciortino, S. 2003, A\&A, 397, 611

Flaccomio, E., Micela, G., Sciortino, S., et al. 2005, ApJS, 160, 450

Glassgold, A. E., Najita, J., \& Igea, J. 1997, ApJ, 480, 344

Glassgold, A. E., Najita, J., \& Igea, J. 2004, ApJ, 615, 972

Glassgold, A. E., Najita, J. R., \& Igea, J. 2007, ApJ, 656, 515

Glassgold, A. E., Meijerink, R., \& Najita, J. R. 2009, ApJ, 701, 142

Gorti, U., \& Hollenbach, D. 2004, ApJ, 613, 424

Gorti, U., \& Hollenbach, D. 2008, ApJ, 683, 287

Gorti, U., Dullemond, C. P., \& Hollenbach, D. 2009, ApJ, 705, 1237

Gregory, S. G., Wood, K., \& Jardine, M. 2007, MNRAS, 379, L35

Güdel, M., Telleschi, A., Audard, M., et al. 2007, A\&A, 468, 515

Henning, T., Semenov, D., Guilloteau, S., et al. 2010, ApJ, 714, 1511
Hollenbach, D., \& Gorti, U. 2009, ApJ, 703, 1203

Igea, J., \& Glassgold, A. E. 1999, ApJ, 518, 848

Imanishi, K., Koyama, K., \& Tsuboi, Y. 2001, ApJ, 557, 747

Kamp, I., Tilling, I., Woitke, P., Thi, W., \& Hogerheijde, M. 2010, A\&A, 510, A18

Koyama, K., Maeda, Y., Ozaki, M., et al. 1994, PASJ, 46, L125

Lennon, M. A., Bell, K. L., Gilbody, H. B., et al. 1988, J. Phys. Chem. Ref. Data, 17,1285

Maloney, P. R., Hollenbach, D. J., \& Tielens, A. G. G. M. 1996, ApJ, 466, 561

Meijerink, R., \& Spaans, M. 2005, A\&A, 436, 397

Meijerink, R., Glassgold, A. E., \& Najita, J. R. 2008, ApJ, 676, 518

Nomura, H., Aikawa, Y., Tsujimoto, M., Nakagawa, Y., \& Millar, T. J. 2007, ApJ, 661, 334

Pravdo, S. H., Feigelson, E. D., Garmire, G., et al. 2001, Nature, 413, 708

Shu, F. H., Najita, J. R., Shang, H., \& Li, Z. 2000, Protostars and Planets IV, 789

Shull, J. M., \& van Steenberg, M. E. 1985, ApJ, 298, 268

Stelzer, B., \& Neuhäuser, R. 2001, A\&A, 377, 538

van der Werf, P. P., Isaak, K. G., Meijerink, R., et al. 2010, A\&A, 518, L42

Verner, D. A., \& Yakovlev, D. G. 1995, A\&AS, 109, 125

Woitke, P., Kamp, I., \& Thi, W. 2009, A\&A, 501, 383

Woitke, P., Pinte, C., Tilling, I., et al. 2010, MNRAS, 405, L26

Wolk, S. J., Harnden, Jr., F. R., Flaccomio, E., et al. 2005, ApJS, 160, 423

Woods, P. M., \& Willacy, K. 2009, ApJ, 693, 1360 